sults of $A_{\max } \simeq 0.4-0.6{ }^{11,12}$ In conclusion, reflectance measurements and plasma-simulation calculations exhibit similarities unexpected only a short time ago.

One of us (R.P.G.) acknowledges receipt of a Fulbright grant.

Note added.--Since the original submission of this paper a related work has appeared. ${ }^{13}$

(a) Guest Researcher, on leave from Los Alamos Scientific Laboratory, Los Alamos, N. Mex. 87544.

${ }^{1}$ C. G。 M。 van Kessel, J. N. Olsen, P. Sachsenmaier, R. Sigel, K. Eidmann, and R. P. Godwin, Projektgruppe für Laserforschung, Max-Planck-Gesellschaft zur Förderung der Wissenschaften, Laboratory Report No. IPP IV/94, 1976 (unpublished).

${ }^{2}$ R. A. Haas, W. C. Mead, W. L. Kruer, D。W. Phillion, H. N. Kornblum, J。 D。 Lindl, D。 MacQuigg, V. C. Rupert, and K. G. Tirsell, Phys. Fluids 20, 322 (1977).

${ }^{3}$ V. M。 Cottles and D。 V. Giovanielli, LASL Report No. LA-UR-77-1096, 1977 (unpublished).

${ }^{4} \mathrm{~V}$. L. Ginzburg, The Propagation of Electromagnetic
Waves in Plasmas (Pergamon, New York, 1964), p。 213. ${ }^{5}$ J. P. Freidberg, R. W. Mitchell, R. L. Morse, and L. I. Rudsinski, Phys。Rev。Lett。28, 795 (1972)。

${ }^{6}$ R. P. Godwin, Phys. Rev。 Lett。28, 85 (1972).

${ }^{7}$ J. S. Pearlman, J. J. Thomson, and C. E. Max, Phys. Rev。 Lett. 38, 1397 (1977).

${ }^{8}$ B. H。 Ripin, Appl. Phys。 Lett. 30, 134 (1977).

${ }^{9}$ R. Ulbricht, Elektrotechn. Z. 21, 595 (1900); see also L. Bergmann and C. Schaefer, Lehrbuch der Experimentalphysik (de Gruyter, Berlin, 1974), Band III, p. 629.

${ }^{10}$ R. Sigel, K. Eldmann, H. C. Pant, and P. Sachsenmaier, Phys。Rev. Lett. 36, 1369 (1976).

${ }^{11}$ W. L. Kruer, R. A. Haas, W. C. Mead, D. W. Phil-

lion, and V。C.Ruppert, in Plasma Physics-Nonlinear Theory and Experiments, Nobel Symposium 36, edited by $\mathrm{H}$. Wilhelmsson (Plenum, New York, 1977).

${ }^{12}$ B. Bezzerides, D。 F。 Du Bois, D. W. Forslund, J. M. Kindel, K. Lee, and E. L. Lindman, in Proceedings of the Sixth International Conference on Plasma Physics and Controlled Nuclear Fusion Research, Berchtesgaden, West Germany, 1976 (International Atomic Energy Agency, Vienna, 1977), Vol. I, p. 123.

${ }^{13}$ K. R. Manes, V. C. Rupert, J. M. Auerbach, P. Lee, and J. E. Swain, Phys. Rev. Lett. 39, 281 (1977).

\title{
Universal Jump in the Superfluid Density of Two-Dimensional Superfluids
}

\author{
David R. Nelson ${ }^{(a)}$ \\ Department of Physics, Harvard University, Cambridge, Massachusetts 02138 \\ and \\ J. M. Kosterlitz \\ Department of Mathematical Physics, University of Birmingham, Birmingham B15 2TT, England
}

(Received 16 September 1977)

\begin{abstract}
We observe that recent theories of phase transitions in the two-dimensional $X Y$ model predict a universal jump in the superfluid density of ${ }^{4} \mathrm{He}$ films as $T_{c}$ is approached from below. Specifically, we find that $\lim _{T \rightarrow T_{c}} \rho_{S}(T) / T=3.52 \times 10^{-9} \mathrm{~g} / \mathrm{cm}^{2}{ }^{\circ} \mathrm{K}$. Analogous results should hold for two-dimensional planar magnets and liquid crystals.
\end{abstract}

A number of theories have been advanced dealing with the critical properties of the classical two-dimensional $X Y$ model. ${ }^{1-6}$ Although the lack of long-range order in such systems has been proven rigorously, ${ }^{7}$ evidence from high-temperature-series expansions ${ }^{8}$ suggests the possibility of a transition at finite temperature into a lowtemperature phase without longe-range order. Indeed, the theories of Refs. 1-6 all predict a low-temperature region which can be characterized as a "phase" of critical points with continuously variable critical exponents. Within this phase, order-parameter correlation functions are expected to fall off at large distances as power laws with temperature-dependent exponents.
There is considerable disagreement, however, on the predictions near the critical temperature $T_{c}$, above which correlations are expected to decay exponentially. Because experimental realizations of two-dimensional $X Y$ behavior may actually be available in ${ }^{4} \mathrm{He}$ films, ${ }^{9}$ in planar magnets, ${ }^{10}$ and in liquid crystals, ${ }^{11}$ it seems desirable to have a simple criterion for determining which of the theories, if any, are correct.

In this Letter, we point out that the theories advanced in Refs. $2 \mathrm{~b}$ and 5 predict a universal jump in the superfluid density of ${ }^{4}$ he films. The size of the jump is related to the critical exponent $\eta\left(T_{c}\right)$, which governs the power-law decay of correlations at the critical temperature. This result 
can be transcribed into analogous predictions for two-dimensional planar magnets and for liquidcrystal films. To the extent we understand Refs. 1,3 , and 4 , these theories would lead to quite different predictions. Because many of the predictions of Wiegmann ${ }^{6}$ agree with the results of Kosterlitz ${ }^{2 \mathrm{~b}}$ and Jose et al., ${ }^{5}$ we expect that the same universal jump in $\rho_{s}(T)$ would result from his theory.

A crucial feature of the theories of Kosterlitz and Thouless ${ }^{2}$ and of Jose $e t a l .^{5}$ is the mapping of a two-dimensional lattice of planar spins onto a neutral Coulomb gas. A Coulomb-gas problem is also suggested by the application of statistical mechanics to a superfluid ${ }^{4} \mathrm{He}$ film. We assume that the relevant probability distribution for the superfluid velocity $v_{s}$ is

$$
P \propto \exp \left\{-\int d^{2} r\left[\frac{1}{2}\left(\rho_{0} / k_{\mathrm{B}} T\right) v_{s}{ }^{2}-m^{2} \ln y_{0}\left|\Delta \times \overrightarrow{\mathrm{v}}_{s}\right|^{2} /(2 \pi \hbar)^{2}\right]\right\},
$$

where, in addition to the usual kinetic-energy term, we have included a term proportional to the total squared vorticity in the fluid, or "enstrophy." As pointed out by Kraichnan, ${ }^{12}$ conservation of "enstrophy" is a feature peculiar to two-dimensional hydrodynamics, and leads to the modified statisticalmechanical description implied by (1). The parameter $\ln y_{0}$ is the thermodynamically conjugate variable to the enstrophy, and was introduced in a rather ad hoc fashion for planar magnets in Ref. 5 . We shall assume that $y_{0}$ is less than unity.

We now decompose the superfluid velocity field into a potential flow term and a part due to vortices:

$$
\overrightarrow{\mathrm{v}}_{s}(\overrightarrow{\mathrm{r}})=(\hbar / m) \nabla \varphi(\overrightarrow{\mathrm{r}})+(2 \pi \hbar / m)(\hat{z} \times \nabla) \int d^{2} \boldsymbol{r}^{\prime} n\left(\overrightarrow{\mathrm{r}}^{\prime}\right) G\left(\overrightarrow{\mathrm{r}}, \overrightarrow{\mathrm{r}}^{\prime}\right)
$$

where $\varphi(\overrightarrow{\mathrm{r}})$ is a smoothly varying function, $n(\overrightarrow{\mathrm{r}})$ is an integer-valued vorticity field, and $m$ is the mass of a helium atom. The unit vector $\hat{z}$ is perpendicular to the plane of the film, $\nabla$ represents a three-dimensional gradient, and the Green's function $G\left(\overrightarrow{\mathrm{r}}, \overrightarrow{\mathrm{r}}^{\prime}\right)$ satisfies

$$
\left[\left(\partial^{2} / \partial x^{2}\right)+\left(\partial^{2} / \partial y^{2}\right)\right] G\left(\overrightarrow{\mathbf{r}}, \overrightarrow{\mathbf{r}}^{\prime}\right)=\delta^{(2)}\left(\overrightarrow{\mathbf{r}}-\overrightarrow{\mathbf{r}}^{\prime}\right) \text {. }
$$

Although we have used a convenient continuum notation, we shall, in fact, impose an ultraviolet cutoff, and require in particular that the logarithmic interaction between two vortices cease at separations smaller than some core radius $a$. On substituting (2) into (1), we find a probability distribution of the form $P \propto e^{\bar{H}}$, with

$$
\bar{H}=-\frac{1}{2} K \int d^{2} r(\nabla \varphi)^{2}+\pi K \iint_{\left|\overrightarrow{\mathrm{r}}-\overrightarrow{\mathrm{r}}^{\prime}\right|>a} \frac{d^{2} \boldsymbol{r}}{a^{2}} \frac{d^{2} r^{\prime}}{a^{2}} n(\overrightarrow{\mathrm{r}}) n\left(\overrightarrow{\mathrm{r}}^{\prime}\right) \ln \frac{\left|\overrightarrow{\mathrm{r}}-\overrightarrow{\mathrm{r}}^{\prime}\right|}{a}+\ln y \int \frac{d^{2} r}{a^{2}} n^{2}(\overrightarrow{\mathrm{r}}),
$$

where $K=\rho_{0} \hbar^{2} / m^{2} k_{\mathrm{B}} T$ and $y=y_{0} e^{-c K}$. In deriving (4), we have approximated $G\left(\overrightarrow{\mathrm{r}}, \overrightarrow{\mathrm{r}}^{\prime}\right)$ by $(1 / 2 \pi) \ln \left(\left|\overrightarrow{\mathrm{r}}-\overrightarrow{\mathrm{r}}^{\prime}\right| /\right.$ $a)+C$, where $C$ is a positive constant related to the core energy whose precise value is unimportant. The kinetic-energy term is finite, provided that we allow only those vorticity complexions with $\int d^{2} r n(\overrightarrow{\mathrm{r}})$ $=0$. Equation (4) is, of course, precisely the spin-wave Coulomb-gas Hamiltonian found for magnetic systems in Refs. 2 and 5. To calculate correlation functions with (4), one must average over smoothly varying velocity fields determined by $\varphi(\overrightarrow{\mathrm{r}})$, and over distinct vorticity complexions determined by $n(\overrightarrow{\mathrm{r}})$. At low temperatures (or small $y_{0}$ ), we expect that $n(\overrightarrow{\mathrm{r}})$ will be zero nearly everywhere, and \pm 1 at isolated points. The vortex-interaction part of (4) is just that proposed years ago by Onsager. ${ }^{13}$ We refer readers to Ref. 12 for a discussion of the relation between continuous and discrete vorticity in classical hydrodynamics.

We shall demonstrate the universality of $\rho_{s}(T) / T$ by calculating this quantity directly from (4). This will be done by using a renormalization transformation to relate a difficult calculation in the presence of vortices to a more tractable one in a vortex-free environment. The superfluid density can be expressed in terms of a correlation function ${ }^{13,14}$ :

$$
K_{R}^{-1} \equiv m^{2} k_{\mathrm{B}} T / \hbar^{2} \rho_{s}(T)=\left(m^{2} / \hbar^{2}\right) \int d^{2} r\left\langle\overrightarrow{\mathrm{v}}_{s}(\overrightarrow{\mathrm{r}}) \circ \overrightarrow{\mathrm{v}}_{s}(\overrightarrow{0})\right\rangle \text {. }
$$

By substituting the velocity decomposition (2) into (5), we can easily derive a formal expression for $K_{R}^{-1}$ as a power series in $y$ :

$$
K_{R}^{-1}=K^{-1}+4 \pi^{3} y^{2} \int_{a}^{\infty}(d r / a)(r / a)^{3-2 \pi K}+O\left(y^{4}\right),
$$

where we have made use of the relation ${ }^{5}\langle n(\overrightarrow{0}) n(\overrightarrow{\mathrm{r}})\rangle=-2 y^{2} r^{-2 \pi K}+O\left(y^{4}\right)$. To this order, only vortex pairs 
with quantum numbers \pm 1 need be taken into account. An expression similar to (6) was obtained in Ref. $5,{ }^{15}$ where a very different (and much more complicated) correlation function was considered. In Ref. 5 , it was shown that, by integrating over length scales between $a_{0}$ and $a_{0} e^{l}$, the series (6) can be rewritten,

$$
K_{R}^{-1}=K^{-1}(l)+4 \pi^{3} y^{2}(l) \int_{a}^{\infty}(d r / a)(r / a)^{3-2 \pi K(l)}+O\left(y^{4}(l)\right),
$$

where $K(l)$ and $y(l)$ obey differential recursion relations, ${ }^{2,5}$

$$
\begin{aligned}
& d K^{-1}(l) / d l=4 \pi^{3} y^{2}(l), \\
& d y(l) / d l=[2-\pi K(l)] y(l),
\end{aligned}
$$

subject to the initial conditions $K(l=0)=K$ and $y(l=0)=y$. Comparing Eqs. (6) and (7), we arrive at an important relation between $K_{R}$ calculated with the bare parameters $K$ and $y$, and $K_{R}$ calculated with the partially "dressed" quantities $K(l)$ and $y(l)$, namely,

$$
K_{R}^{-1}(K, y)=K_{R}^{-1}(K(l), y(l)) \text {. }
$$

A direct determination of $K_{R}^{-1}(K, y)$ from the series (6) can be difficult, especially for $2 \pi K \gtrsim 4$, where the integrals appearing in (6) are poorly convergent. Equation (9), however, relates such a calculation to one with altered parameters $K(l)$ and $y(l)$. For $T \leqslant T_{c}$, the differential equations (8) drive $y(l)$ to zero ${ }^{2,5}$, i.e.,

$$
\lim _{l \rightarrow \infty} y(l)=0 \text {. }
$$

This suggests that (9) can be exploited below $T_{c}$ by taking the limit $l \rightarrow \infty$,

$$
\begin{aligned}
K_{R}^{-1}(K, y) & =\lim _{l \rightarrow \infty} K_{R}^{-1}(K(l), y(l)) \\
& =\lim _{l \rightarrow \infty} K^{-1}(l)\left(T \lesssim T_{c}\right),
\end{aligned}
$$

where we have made use of (7) with $y(l)=0$ 。 The critical temperature is identified ${ }^{2 b, 5}$ with the largest temperature such that

$$
\lim _{l \rightarrow \infty} y(l)=0 \text { 。 }
$$

As $T$ goes to $T_{c}$ from below, it is easily shown from Eqs. (8) that

$$
\lim _{T \rightarrow T_{c}^{-}} K_{R}^{-1}=\lim _{T \rightarrow T_{c}^{-}} m^{2} k_{\mathrm{B}} T / \hbar^{2} \rho_{s}(T)=\frac{1}{2} \pi .
$$

Thus, $m^{2} k_{\mathrm{B}} T / \hbar^{2} \rho_{s}(T)$ approaches a universal constant as $T \rightarrow T_{c}{ }^{-}-$a constant which is independent of the initial values of $K$ and $y$.

It is instructive to produce plots of $\rho_{s}(T)$ vs $T$ for the Coulomb-gas model (4) by integrating the recursion relations (8) numerically until $y(l) \simeq 0$. We have done this for different values of $\rho_{0}$, which could be varied in an experiment by, say, changing the thickness of the ${ }^{4} \mathrm{He}$ film. Although the model produces a series of flat curves without much structure in $\rho_{s}(T)$ below $T_{c}$, there is a characteristic square-root $\operatorname{cusp}^{16}$ as $T \rightarrow T_{c}{ }^{-}$, which depresses $\rho_{s}\left(T_{c}\right)$ by about $10 \%$ of its value at $T=0$. Of course, in a real film, many other processes will tend to depress $\rho_{s}(T)$ below its $T$ $=0$ value; however, we expect that an additional $10 \%$ effect due to the cusp will still be important. The various values of $\rho_{s}\left(T_{c}\right)$ and $T_{c}$ all lie on a straight line through the origin with slope $2 m^{2} k_{\mathrm{B}} /$ $\hbar^{2} \pi=3.52 \times 10^{-9} \mathrm{~g} / \mathrm{cm}^{2}{ }^{\circ} \mathrm{K}$. We predict that this slope will be a universal constant for ${ }^{4} \mathrm{He}$ films despite the fact that $\rho_{s}\left(T_{c}\right)$ and $T_{c}$ will depend on coverage, the details of the wall potential, etc. Although the amplitude of the square-root cusp is nonuniversal, we can estimate its size from our model calculations. Near $T_{c}$, we find $\rho_{s}(T) / T$ $\approx\left[\rho_{s}\left(T_{c}\right) / T_{c}\right]\left[1+0.50\left(1-T / T_{c}\right)^{1 / 2}+O\left(T_{c}-T\right)\right]$. Presumably, $\rho_{s}(T)$ is zero above $T_{c}$ 。

The universal result (10) can be couched in terms of a prediction for the critical exponent $\eta(T)$ as $T \rightarrow T_{c}{ }^{-}$. Since it is an immediate consequence of the theories of Refs. $2 b$ and 5 that

$$
\eta(T)=\lim _{l \rightarrow \infty}[1 / 2 \pi K(l)],
$$

we have $\eta(T)=m^{2} k_{\mathrm{B}} T / 2 \pi \hbar^{2} \rho_{s}(T)$, which gives $\eta=\frac{1}{4}$ at $T_{c}$. (There are actually logarithmic corrections to the power-law decay of correlations at $T_{c},{ }^{2 \mathrm{~b}, 6}$ but these need not concern us here.) An identical relation between $\eta$ and $\rho_{s}$ appears to us to be a consequence of the theories of Refs. 1, 3, and 4 . Since Zittartz ${ }^{3}$ predicts transition at $T_{c}$ 's such that $\eta\left(T_{c}\right)=2$ and 4 , and Luther and Scalapino ${ }^{4}$ predict a nonuniversal $\eta\left(T_{c}\right)$ [in particular, $\eta\left(T_{c}\right)$ can assume the value $1 / \sqrt{8}]$, precision experimental measurements of $\rho_{s}(T)$ in ${ }^{4} \mathrm{He}$ films could presumably decide between the various theories. Berezinskii ${ }^{1}$ predicts that $\rho_{s}(T)$ vanishes at $\boldsymbol{T}_{c}$, which can also be checked experimentally. Meas urements of third-sound propagation in films $\mathrm{s}^{17}$ are not inconsistent ${ }^{16}$ with the prediction (10).

Predictions for the spin-wave stiffness in twodimensional magnetic systems analogous to (10) follow by making the standard analogy between $X Y$ magnetism and superfluidity. ${ }^{18}$ Two-dimensional nematic crystals can be described by a 
free-energy density of the form ${ }^{11}$

$$
F=\frac{1}{2} K_{1}(T)[\nabla \cdot \overrightarrow{\mathrm{n}}(\overrightarrow{\mathrm{r}})]^{2}+\frac{1}{2} K_{3}(T)|\overrightarrow{\mathrm{n}}(\overrightarrow{\mathrm{r}}) \times[\nabla \times \overrightarrow{\mathrm{n}}(\overrightarrow{\mathrm{r}})]|^{2},
$$

where $\vec{n}(\vec{r})$ is a two-dimensional director field. The $K_{2}$ Franck coefficient corresponding to "twist" distortions is absent for $d=2$. A renormalizationgroup analysis by Nelson and Pelcovits ${ }^{19}$ has shown that deviations from the one-Franck-constant approximation $K_{1}=K_{3}$ are irrelevant variables in the low-temperature $X Y$-model phase characterized by power-law decay of correlations. In this limit, Eq. (11) reduces to a continuum version of the two-dimensional $X Y$ model. ${ }^{11}$ Taking over the analysis for superfluids, we find

$$
\lim _{T \rightarrow T_{c^{-}}} K_{i}(T) / k_{\mathrm{B}} T=2 / \pi, i=1,3 .
$$

De Gennes ${ }^{11}$ has suggested that a nematic film floating on the surface of a fluid may give a good realization of a liquid-crystal system described by (11). "Soap-bubble" films of a small number of smectic liquid-crystal layers may also provide such an experimental realization. ${ }^{20}$

Although we have demonstrated explicitly only that $\rho_{s}(T) / T$ should approach a universal constant for the model defined by (4), we believe our conclusions remain unchanged when other irrelevant variables are taken into account. Indeed, if we denote by $\left\{y_{i}\right\}$ a collection of additional perturbations representing, say, vortices with quantum numbers $\pm 2, \pm 3, \ldots$, or interactions between vortices and the field $\varphi(\vec{r})$, it seems clear that the renormalization groups constructed in Refs. $2 \mathrm{~b}$ and 5 would lead to a simple generalization of (9): $K_{R}^{-1}\left(K, y,\left\{y_{\mathbf{i}}\right\}\right)=K_{R}^{-1}\left(K(l), y(l),\left\{y_{i}(l)\right\}\right)$. In $d$ dimensions this result reads $K_{R}\left(K, y,\left\{y_{i}\right\}\right)$ $=e^{(2-d) l} K_{R}\left(K(l), y(l),\left\{y_{i}(l)\right\}\right)$, which is just the Josephson scaling relation ${ }^{21}$ in disguise. It is easy to show using the methods of Refs. 2 and 5 that the perturbations $\left\{y_{i}(l)\right\}$ as well as $y(l)$ all tend to zero as $l \rightarrow \infty$ in the low-temperature phase of the two-dimensional $X Y$ model。An analysis in the limit $l \rightarrow \infty$ then leads immediately to the universal result (10).

It is worth noting that the universal (minimum) value of $\rho_{s}(T) / T$ which is approached as $T \rightarrow T_{c}$ bears a striking resemblance to the predicted ${ }^{c 2}$ minimum conductivity in two-dimensional metals.

This investigation was stimulated in part by experiments of D. Bishop and J. D. Reppy on ${ }^{4} \mathrm{He}$ films. We are grateful to them for discussions of the experimental situation, as well as to $R$. B.
Meyer and the members of the liquid-crystal group at Harvard University for similar discussions. Interesting conversations with $\mathrm{B}$ 。 I. Halperin and $\mathrm{P}$ 。C. Martin are also acknowledged. One of us (D.R.No) would like to acknowledge stimulating discussions with $\mathrm{P}$ 。C. Hohenberg during the preliminary stages of this investigation, as well as support by the National Science Foundation through Grant No. DMR 77-10210AO3. One of us (J.M.K.) would like to acknowledge the hospitality of Harvard University, where part of this work was carried out。

\footnotetext{
(a) Work supported by a Junior Fellowship, Harvard Society of Fellows.

${ }^{1}$ V. L. Berezinskii, Zh. Eksp. Teor. Fiz. 59, 907 (1970) [Sov. Phys. JETP 32, 493 (1971)]; V. L. Berezin-
} skii, Zh. Eksp. Teor. Fiz. 61, 1144 (1971) [Sov. Phys. JETP 34,610 (1971).]

${ }^{2 a}$ J. M. Kosterlitz and D. J. Thouless, J. Phys. C $\underline{6}$, 1181 (1973).

${ }^{2 b}$ J. M. Kosterlitz, J. Phys. C ㄱ, 1046 (1974).

${ }^{3}$ J. Zittartz, Z. Phys. 23B, 55, 63 (1976).

${ }^{4}$ A. Luther and D. J. Scalapino, Phys. Rev. B $\underline{16}$, 1153 (1977).

${ }^{5}$ J. V. José, L. P. Kadanoff, S. Kirkpatrick, and D. R. Nelson, Phys. Rev. B 16, 1217 (1977).

${ }^{6} \mathrm{P}$. G. Wiegmann, to be published.

${ }^{7}$ See, e.g., P. C. Hohenberg, Phys. Rev. 158, 363 (1967); N. D. Mermin J. Math. Phys. (N.Y.) $\underline{8}, 1061$ (1967).

${ }^{8}$ H. E. Stanley and T. A. Kaplan, Phys. Rev. Lett. 17 , 913 (1966). See also, W. J. Camp and J. P. Van Dyke, J. Phys. C $\underline{8}, 336$ (1975).

${ }^{9}$ See, e.g., S. Doniach, Phys. Rev. Lett. 31,1450 (1973).

${ }^{10}$ See, e.g., R. Narvarro and J. L. de Jongh, Physica (Utrecht) 84B, 229 (1976).

${ }^{11}$ P. G. de Gennes, The Physics of Liquid Crystals (Oxford Univ. Press, London, 1974), p. 111.

${ }^{12}$ R. H. Kraichnan, J. Fluid. Mech. 67, 155 (1975). ${ }^{13}$ L. Onsager, Nuovo Cimento Suppl. 6 , 279 (1949); see also, R. P. Feynmann, Prog. Low Temp. Phys. 1 , (1955).

${ }^{14}$ P. C. Hohenberg and P. C. Martin, Ann. Phys. $\underline{34}$, 291 (1965).

${ }^{15}$ See Eq. (5.1) of Ref. 5. Here $K_{R}$ was called $K_{\text {eff }}$.

There is a factor of 2 error in the coefficient of the integral in this equation.

${ }^{16} \mathrm{~J}$. M. Kosterlitz and D. J. Thouless, to be published. An estimate of the jump in $\rho_{s}(T)$ was made in this reference but it was not realized that results such as (10) should be universal.

${ }^{17}$ K. R. Atkins and I. Rudnick, Prog. Low Temp. Phys. 6,37 (1970).

${ }^{18}$ B. I. Halperin and P. C. Hohenberg, Phys. Rev. $\underline{188}, 898$ (1969). 


\begin{tabular}{ll}
\hline${ }^{19}$ D. R. Nelson and R. A. Pelcovits, Phys. Rev. B 16, & ${ }^{21}$ B. D. Josephson, Phys. Lett. $21,608(1966)$. \\
& ${ }^{22}$ D. C. Licciardello and D. J. Thouless, Phys. Rev. \\
${ }^{20}$ R. B. Meyer, private communication. & Lett. $\underline{35}, 1475$ (1975).
\end{tabular}

\title{
Unusual Phonon Heat Transport in an Amorphous Superconducting PbCu Film
}

\author{
H. v. Löhneysen ${ }^{(a)}$ and F. Steglich \\ II. Physikalisches Institut der Universität zu Köln, D-5 Köln, West Germany
}

(Received 11 July 1977)

\begin{abstract}
We report thermal conductivity measurements of a $\mathrm{Pb}_{0.9} \mathrm{Cu}_{0.1}$ film before (amorphous) and after (crystalline) annealing. Below $T_{c}$, where electronic effects are negligible, we found that the phonon contribution in the amorphous phase $k_{\text {am }}^{\text {ph }}$ was much larger than that in the crystalline phase. We also found that the temperature dependence of $k_{\text {am }}{ }^{\text {ph }}$ resembles that of amorphous dielectrics, thus providing evidence that this sort of "glassy" behavior does not require the presence of covalent bonds.
\end{abstract}

Amorphous insulators like glasses, polymers, and $a$-Se show distinctive thermal properties below $1 \mathrm{~K}$. For these substances contributions to the specific heat proportional to $T$ and $T^{3}$ are observed in addition to the Debye term. ${ }^{1}$ Furthermore, a $T^{2}$ variation of the (phonon) thermal conductivity is found in contrast to the $T^{3}$ dependence seen in crystals." This "glassy" behavior, also visible in the $k$ vs $T$ curves of semiconducting $a-\mathrm{Ge}^{2}$ can be successfully interpreted in terms of localized low-energy excitations (LEE) as assumed in the Anderson-Halperin-Varma-Phillips tunneling model. ${ }^{3,4}$ To date, however, this model lacks a microscopic explanation. Thus, since all the mentioned materials are covalently bonded, it is not clear whether the amorphous structure itself or the presence of covalent bonds in a disordered environment is essential for "glassy" behavior.

Amorphous metals, of course, are the natural probe for such an investigation. Very recently, a phonon thermal conductivity $k^{\mathrm{ph}} \sim T^{2}$ was reported for several bulk metallic glasses. ${ }^{5}$ However, all of these materials, most of which contain metalloids with covalent bonds, are in the normal state (including $\mathrm{Ti}_{0.5} \mathrm{Be}_{0.4} \mathrm{Zr}_{0.1}$ which becomes superconducting only below $0.32 \mathrm{~K}$ ). In addition, they all contain transition metals which can act as resonance scattering centers of the conduction electrons. Therefore, the influence of the conduction electrons (as heat carriers as well as phonon scatters) is not easy to calculate, and as a result conclusions drawn for $k^{\mathrm{ph}}$ are, at most, only of qualitative value. To avoid these difficulties, an amorphous superconducting $\mathrm{Pb}_{0.9} \mathrm{Cu}_{0.1}$ $(P b C u)$ film, prepared by quench condensation from the vapor phase, was chosen for the present investigation: Well below $T_{c}=6.5 \mathrm{~K}$, the influence of the few remaining electronic quasiparticles on $k$ is negligible. Besides, in $\mathrm{PbCu}$ there are no constituents with covalent bonds.

Another motivation for thermal conductivity measurements on amorphous metal films stems from the fact that in quench-condensed crystalline metal films $k^{\mathrm{ph}}$ is almost negligible as compared to the electronic contribution $k^{e} \cdot 6.7$ It has been argued ${ }^{6}$ that in this case the phonons are scattered strongly from spatially extended lattice defects, i.e., stacking faults, dislocations, and grain boundaries. However, since these defects should be absent in a solid without longrange order, we hoped to observe a substantial recovery of the ratio $k^{\mathrm{ph} / k^{e}}$ in amorphous (as compared to crystalline) $\mathrm{PbCu}$. This should enable us to use $k^{\text {ph }}$ as a probe of "glassy" behavior.

The amorphous film was prepared by means of the Drehrohrofen technique deveoped by Hilsch and Martienssen ${ }^{8}$ : A mechanically homogenized 90-at.\% $\mathrm{Pb}, 10$-at.\% Cu mixture was evaporated in small pellets (each producing less than one atomic layer) onto a cooled substrate. As substrate we used a 12.5- $\mu \mathrm{m}$-thick circular Kapton foil, which sealed an additional $\mathrm{Cu}$ tank clamped to the cold part of a $\mathrm{He}^{3}$ cryostat. ${ }^{9}$ During evaporation of the metal film, this $\mathrm{Cu}$ tank was filled with $\mathrm{He}^{4}$ exchange gas (5 Torr). Thus the substrate temperature could be kept below $15 \mathrm{~K}$, i.e., well below the crystallization temperature $(30 \mathrm{~K})$ of $\mathrm{PbCu}$. The pressure in the cryostat remained below $10^{-6}$ Torr during evaporation. Afterwards the $\mathrm{He}^{4}$ exchange gas was pumped out and the total thermal conductance $K_{T}$ of the sub- 